\title{
Facilitation of variable interval performance by intermittent conditioned reinforcement
}

\author{
E. F. MALAGODI \\ UNIVERSITY OF MIAMI, FLORIDA
}

Three rats were placed on either multiple or mixed schedules of VI $2 \mathrm{~min}$. primary reinforcement. Conditioned reinforcement was superimposed in one of two components on a VI $30 \mathrm{sec}$. schedule. Response rates in these components increased above response rates in the components without the added schedule of conditioned reinforcement.

Several studies have recently reported facilitative effects of intermittent conditioned reinforcement (Sr) upon behavior maintained by intermittent primary reinforcement. Findley \& Brady (1965) found shorter worktime and shorter latencies before response initiation in a multiple schedule component that featured an $\mathrm{S}^{r}$ presented on fixed ratio (FR) 400 when the schedule of primary reinforcement was FR 4000. Randolph \& Sewell (1965) added an FR 10 schedule of Sr to differential reinforcement of low rate (DRL) schedules of primary reinforcement. They found small and transitory increases in response rates in two of three cases.

In the present study baseline performance generated by a variable interval (VI) schedule of food reinforcement was selected in an attempt to demonstrate reliable and durable increases in overall rates of responding as a function of a superimposed schedule of conditioned reinforcement.

\section{Subjects and Apparatus}

Three experimentally naive male hooded rats, approximately 120 days of age at the start of the experiment, were maintained at $80 \%$ to $85 \%$ of their freefeeding weights.

A standard Grason-Stadler experimental chamber was enclosed within a ventilated, sound-attenuating ice box. Automatic programming and recording equipment were located in an adjacent room.

Procedure

Rat R-1 was placed on a multiple VI $2 \mathrm{~min}$. VI $2 \mathrm{~min}$. food schedule after several sessions on continuous reinforcement. The discriminative stimuli $\left(\mathrm{SD}^{\mathrm{D}}\right)$ accompanying the multiple components were the presence and absence of a low-intensity, low-frequency clicker. The two stimulus conditions alternated with each 15 primary reinforcement deliveries for a total of 60 or 75 food pellets per session. The $\mathrm{Sr}$ stimuli were introduced after 11 sessions on the multiple VI $2 \mathrm{~min}$. VI 2 min. program. A 1.0 sec. presentation of the house-light accompanied food delivery in the presence of the clicker $\mathrm{SD}$. In the absence of the clicker a $1.0 \mathrm{sec}$. presentation of a high-frequency, moderateintensity buzzer accompanied food delivery; in addition, the buzzer presentations occurred on an overall schedule of VI 30 sec. in such a manner that each fourth buzzer presentation accompanied food delivery. The component at the start of each session was alternated from day to day.

After 16 sessions on the above program, the buzzer and house-light schedules were reversed. The buzzer, in the absence of the clicker $\mathrm{S}^{\mathrm{D}}$, now accompanied food delivery on VI $2 \mathrm{~min}$. The house-light, in the presence of the clicker $\mathrm{SD}$, was scheduled on VI $30 \mathrm{sec}$. with every fourth light presentation accompanying food delivery.

Rats $X-2$ and $X-3$ were trained in a similar manner, without the clicker- no-clicker arrangement. The absence of continuous $\mathrm{S}^{\mathrm{D}}$ accompanying schedule components characterizes a mixed schedule. These Ss received buzzer or house-light presentations with food delivery (alternating in blocks of 15 reinforcements) from the first day of training through nine sessions of VI $2 \mathrm{~min}$. reinforcement before the added VI $30 \mathrm{sec}$. schedules of $\mathrm{S}^{r}$ were superimposed. Then, rat X-2 received the buzzer on VI 30 sec.; the house-light continued to accompany food on VI $2 \mathrm{~min}$., in the component in which the house-light was scheduled. Rat X-3 received the house-light on VI 30 sec.; the buzzer continued to accompany food on VI $2 \mathrm{~min}$., in the component in which the buzzer was scheduled. No schedule reversals were attempted for these Ss. Results

Figure 1 presents the relative response rates in the components with the added VI 30 sec. $\mathrm{S}^{r}$ schedules. The data for rat $\mathrm{R}-1$ indicate that consistently higher response rates in the VI $30 \mathrm{sec}$. buzzer components did not emerge until after several sessions with the added $\mathrm{s}$ schedule. The mean relative response rate was .77 for the last 10 sessions of VI 30 sec. buzzer scheduling. The reversal of buzzer and light schedules produced smaller differences in response rates, although the differences emerged on the first day of

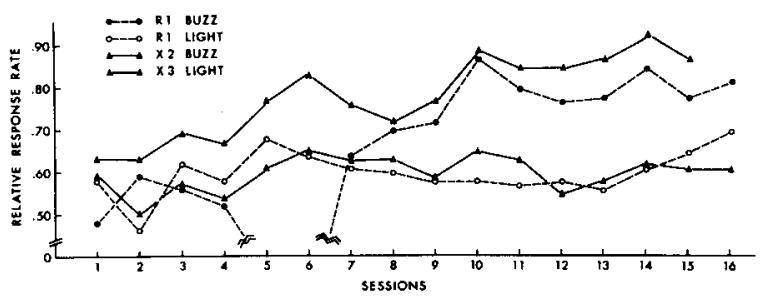

Fig. 1. Relative response rates in components with added VI 30 sec. schedules of conditioned reinforcement. 


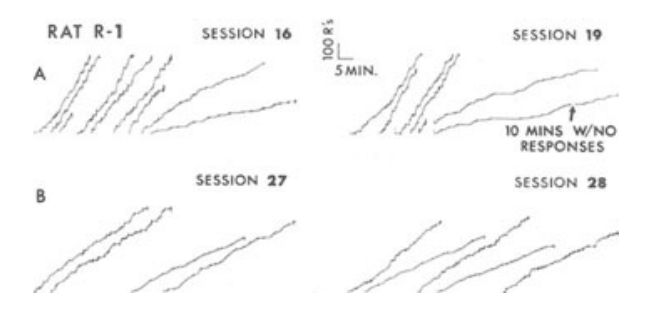

Fig. 2. Representative cumulative records of rat $R-1$. Segment identification is given in the text.

reversal. The mean relative response rate was .60 for all sessions of VI 30 sec. light scheduling.

The data for rats $\mathrm{X}-2$ and $\mathrm{X}-3$ also show consistently higher response rates in the components with the added $\mathrm{S}^{\mathbf{r}}$ schedule. The mean relative response rate for rat $\mathrm{X}-2$ was .78 in the VI 30 sec. buzzer component. The mean relative response rate for rat X-3 was .60 in the VI $30 \mathrm{sec}$. light component.

Typical cumulative records from rat $\mathrm{R}-1$ are presented in Fig. 2. In the "A" portion of the figure the segments with pips both above and below the response curve identify the components with the added buzzer presentations; pips above the response curve indicate food delivery, while pips below the response curve identify buzzer presentations. Segments with pips occurring only below the response curve identify the components with light and food scheduled simultaneously on VI $2 \mathrm{~min}$. Examination of these records indicates considerable bursting in the added buzzer components, while long period of no responding characterized behavior in the components without the added $\mathrm{S}^{\mathbf{r}}$ schedule. In the "B," or reversal, portion of the figure, segments with pips above and below the response curve identify components with the added VI 30 sec. house-light schedule; pips above the response curve indicate food delivery, while pips below the response curve indicate house-light presentations. In the segments with pips occurring only below the response curve, the pips identify both buzzer and food scheduled simultaneously on VI $2 \mathrm{~min}$. The segments for session 28 are presented in the order in which they were recorded, and show relatively stable performance in both components with consistently higher overall rates occurring in the VI $30 \mathrm{sec}$. house-light components.

The individual records in Fig. 3 show typical VI performance for rats $X-2$ and $X-3$, with consistently higher response rates in the components featuring the

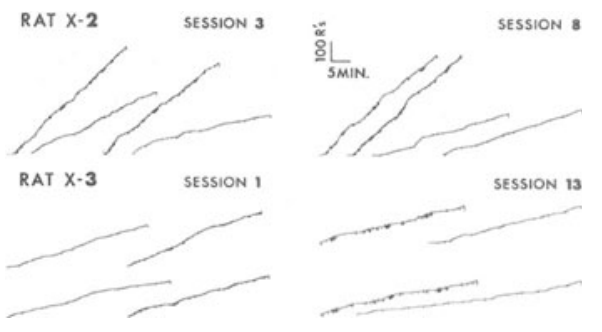

Fig. 3. Representative cumulative records of rats $x-2$ and $x-3$. Segment identification is given in the text.

added $\mathrm{Sr}$ schedule. Segment identification is the same as in Fig. 2. The segments are presented in the order in which they were recorded for rat X-2 during session 3 , and for rat $X-3$ during session 1 . Although the data for rat X-3 indicate little difference between components in total number of responses per segment, the records for session 13 are typical of this S's performance in that the time to complete the components with the added VI $30 \mathrm{sec}$. light schedule is considerably shorter than the time taken to complete the components without the त. d intermittent $\mathrm{s} r$.

. ssion

The results indicate that superimposed schedules of conditioned reinforcement can increase overall response output when the schedule of primary reinforcement allows for such a change. In the multiple ratio schedule employed by Findley \& Brady (1965) a fixed number of responses determined the completion of each component, thereby restricting facilitative effects of the $S^{r}$ to decreases in component worktime. The facilitation of performance can be durable in the VI paradigm as increases in response rates do not decrease primary reinforcement density as was the case with the DRL schedules employed by Randolph \& Sewell (1965).

The stimulus control obtained with rats $\mathrm{X}-2$ and $\mathrm{X}-3$ is similar to the control exerted by brief $\mathrm{S}^{\mathrm{D}}$ in multiple primed schedules of reinforcement (Ferster \& Skinner, 1957).

\section{References}

Ferster, C. B., \& Skinner, B. F. Schedules of reinforcement. New York: Apoleton-Century-Crofts, 1957.

Findley, J. D., \& Brady, J. V. Facilitation of large ratio performance by use of conditioned reinforcement. J. exp. Anal. Behav., 1965, 8, 125-129.

Randolph, J. J., \& Sewell, W. R. Competitive conditioned reinforcement during differential reinforcement of low rates. Psychon. Sci., 1965, 3, 411-412. 